\title{
Comparative Study For Performance ANALYSIS OF VOIP CODECS OVER WLAN IN NON- MOBILITY SCENARIOS
}

\author{
Shreekant Gurrapu ${ }^{1}$,Dr. Saurabh Mehta ${ }^{2}$ and Prof.Shraddha Panbude ${ }^{3}$ \\ $1,2 \& 3$ Department of Electronics and Telecommunication, Vidyalankar institute of \\ Technology, Mumbai India.
}

\begin{abstract}
Voice over IP (VoIP) applications such as Skype, Google Talk, and FaceTime are promising technologies for providing cheaper voice calls to end users over extant networks. Wireless networks such as WiMAX and Wi-Fi focus on providing perfection of service for VoIP. However, there are numerous aspects that affect quality of voice connections over wireless networks [13]. The adoption of Voice over Wireless Local Area Network is on tremendous increase due its relief, non-invasive, economicexpansion, low maintenance cost, universal coverage and basic roaming capabilities. However, expansion Voice over Internet Protocol (VoIP) over Wireless Local Area Network (WLAN) is a challenging task for many network specialist and engineers. Voice codec is one of the most critical components of a VoIP system. In this project, we evaluate the performance analysis of various codecs such as G.711, G.723 and G.729 over Wi-Fi networks. NS2 WiFi simulation models are designed. Performance metrics such as Mean Opinion Score (MOS), average end-to-end latency, and disconcert are evaluated and discussed [13].
\end{abstract}

1. In this paper, our area of interest is to compare and study the performance analysis of VoIP codecs in Non-mobility scenarios by changing some parameters and plotting the graphs throughput, End to end Delay, MOS, Packet delivery Ratio, and Jitter by using Network Simulator version.

2. In this paper we analyze the different performance parameters, Recent research has focused on simulation studies with non- mobility scenarios to analyze different VoIP codecs with nodes up to 5. We have simulated the different VoIP codecs in non-mobility scenario with nodes up to 300.

\section{KEYWORDS:}

Voice over Internet Protocol, Wireless Local Area Network (WLAN), Codecs, throughput, delay.

\section{INTRODUCTION:}

The recent Voice over IP (VOIP) applications such as Skype, Google Talk, and Face Time have changed the way people communicate to each other. Due to the low cost, people find VOIP as an alternative to the expensive traditional Public Switched Telephone Network (PSTN). VOIP has set of parameters that defined its Quality of Service (QoS) such as end to delay, jitter, packets loss, Mean Opinion Score (MOS, and throughput[13]. The existing wireless networks such as WiFi offer flexibility to support such applications. At the time the IEEE 802.11 (Wi-Fi) technology showed great success as cheap wireless internet access. The Motive of this survey paper is to analyse of Qos in VOIP [13]. 


\section{VOIP}

Voice over IP (VoIP) is the real-time application that is probably the most widely-spread on today's networks. I'll provide here some basic facts related to VoIP. Figure below shows the endto-end path as needed for VoIP communication (a similar path exists in the opposite sense for a bi-directional connection). An audio input device, such as a microphone, is required at the sending end. The audio signal is appeared into digital form by an analog-to-digital converter. Due to the packet-switched nature of computer networks, voice data has to be packetized and encoded prior to being transmitted. Encoding (as well as decoding) is done by codecs that transform sampled voice data into a specific network-level representation and back. Most of the codecs are defined by standards of the International Telecommunication Union, the Telecommunication division (ITU-T) [14].

Each of them has different properties regarding the amount of bandwidth it requires but also the comprehend quality of the encoded speech signal. After binary information is encoded and packetized at the sender end, packets enclose voice data can be pass on the network. Voice packets interact in the network with other application packets and are routed through shared relation to their destination. At the receiver end they are decapsulated and decode. Decode may include other steps as well, the most typical being dejittering. Other examples are defect improvement and packet loss niche. The flow of digital data is then converted to analogue form again and played at an output device, usually a speaker [14].

\section{WI-FI}

Wi-Fi is commonly used in residential, business, and public areas. It is notable that the perceived throughput in Wi-Fi does not match the real throughput. Also, all users share the access to the channel which is very critical for all real time traffic in general and especially VoIP. The Wificonnection with low capacity has serious impact on Qos. Beside the high traffic generated by users, both protocols, VoIP and Wi-Fi, create large headers which result in high drawback on VoIP performance [14].

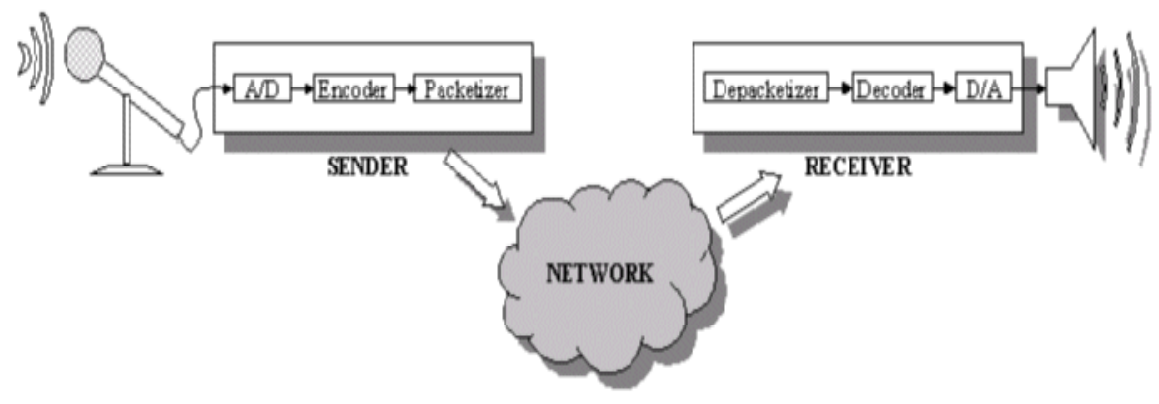

Fig.1 End-to-end data path for VoIP communication[13]

\section{QOS ISSUES OF VOIP APPLICATION}

Quality of Service (QoS) is what determines if a technology can successfully deliver high value services such as voice and video. QoS is referred as the ability to control the mixture of bandwidth, delay, jitter, and packet loss in a network in order to deliver a network service Ensuring high voice call quality over VoIP traffic. QoS for VoIP is defined using different parameters. The BE class which is used for data stream with no support for latency and PDR. In 
this paper, the quality of services for VoIP is measured in terms of end to end latency, jitter and data loss. Latency is defined as the time required for a frame or a packet to travel from the source to its final destination [13].

The main source of delay is categorized into: Devolution Delay and receiver processing delay, efficiency calculation inoperable or inadequate, technological impact, sequencing the packets which outcomes queuing delay etc. An absolute value of delay difference between shortlistedpackets to arrive at receiver is called as jitter. It is not guaranteed that all the packets will follow similar route and confrontation the similar routes to reach the target over the network, and added with the backlogin the network normally resulting in data frames outcomeof order and with shift latency. No disconcert means a network with constant latency and no modification. The amount of data that can actually be pass over the movement channel is called flow capacity. It is used to Estimate the competence of network. The ratio between the quantum of information and the sum of use data, control data and retransmitted data if error is concluded as throughput of a network [10].

Nowadays, people get advantage of the existing data networks by enjoying various ways of communication e.g. text messages, voice calls, and video calls. The traditional phone networks cannot compete with these type of services due to low equipment's and operating cost, and the ability of integrating voice and data in applications. The QoS for VoIP can be measured by evaluating three performance metrics: Mean Opinion Score (MOS), Jitter, and end-to-end delay [18].

\subsection{MEAN OPINION SCORE (MOS):}

MOS is a scale from 1 to 5 which measures the quality of the voice.

Table 1 Mean Opinion Score (MOS)[13]

\begin{tabular}{|c|c|c|}
\hline Quality Scale & Score & Listening effort Scale \\
\hline Excellent & 5 & No effort required \\
\hline Good & 4 & No appreciable effort required \\
\hline Fair & 3 & Moderate effort required \\
\hline Poor & 2 & Considerable effort required \\
\hline Bad & 1 & No meaning understood with reasonable effort \\
\hline
\end{tabular}

\subsection{JITTER:}

The variation in arrival time of consecutive packets is called jitter. In Predecoding, packets should get deliver and some are not deliver in particular size buffered. Jitter should determine by latency of packets over a interval of time.

\subsection{PACKeT END-To-End Delay:}

The amount of packet sent from source to destination which has measured in interval of time.It includes network latency, code and decode delay and data shrinkage. 
Table 1 shows the guidelines for voice quality measurement for both jitter and end-to- end delay as it is provided by ITU Telecommunication Standardization Sector (ITU-T).

Table 2 Guideline for the Voice Quality Measurement [1]

\begin{tabular}{|c|r|r|r|}
\hline Network Parameter & Good & Acceptable & Poor \\
\hline Delay(ms) & $0-150$ & $150-300$ & $>300$ \\
\hline Jitter(ms) & $0-20$ & $20-50$ & $>50$ \\
\hline
\end{tabular}

\subsection{THROUGHPUT:}

The throughput corresponds to the amount of data in bits that is transmitted over the channel per unit time. Generally the throughput measured in bits per second (bps).

\section{Simulation:}

Table 3. Comparison of Various Network Simulators

\begin{tabular}{|c|c|c|c|c|c|}
\hline $\begin{array}{l}\text { Sr } \\
\text { No }\end{array}$ & Parameter & OPNET & OMNeT++ & QualNet & NS2 \\
\hline 1 & License & Commercial & Open Source & Commercial & Open Source \\
\hline 2 & Support & $\begin{array}{c}\text { OPNET has } \\
\text { Integrated, GUI- } \\
\text { based debugging } \\
\text { and analysis for } \\
\text { the users. }\end{array}$ & $\begin{array}{l}\text { OMNeT++ has a } \\
\text { well- designed } \\
\text { simulation engine } \\
\text { and powerful GUI, } \\
\text { Includes Graphical } \\
\text { and command line } \\
\text { interfaces for } \\
\text { simulation } \\
\text { execution In terms } \\
\text { of commercial } \\
\text { simulator, }\end{array}$ & $\begin{array}{l}\text { Qualnet has } \\
\text { complete } \\
\text { GUI provided, }\end{array}$ & $\begin{array}{l}\text { Ns-2 is most } \\
\text { popular } \\
\text { simulator for } \\
\text { academic } \\
\text { research } \\
\text { Graphical as well } \\
\text { as command line } \\
\text { interface }\end{array}$ \\
\hline 3 & Platform & $\begin{array}{c}\mathrm{C}, \mathrm{C}++, \text { Opnet } \\
\text { modeler } \\
\text { software }\end{array}$ & Linux,mac-os,unix & Linux & $\begin{array}{c}\text { Unix,mac-OS } \\
\text { Microsoft } \\
\text { window } \\
\text { Cygwin }\end{array}$ \\
\hline 4 & Specific type & Castalia & Nsrlsensorsim & Viptos & SensorSim \\
\hline 5 & Mobility & Yes & No & No & Yes \\
\hline 6 & $\begin{array}{c}\text { Language } \\
\text { /Programming }\end{array}$ & $\begin{array}{c}\mathrm{C}++/ \text { Java object- } \\
\text { oriented }\end{array}$ & $\begin{array}{c}\mathrm{C}++/ \mathrm{C}++(\text { Highly } \\
\text { portable with } \\
\text { Windows,Linu } \mathrm{x} \\
\text { and Mac OSX }\end{array}$ & $\begin{array}{c}\text { Parsec C++/ } \\
\text { Parsec } \\
\text { (Simulation } \\
\text { language } \\
\text { Derived } \\
\text { from C) }\end{array}$ & $\begin{array}{c}\mathrm{C}++/ \mathrm{OTCL} \\
\text { Object oriented } \\
\text { extension of Tcl } \\
\text { and } \mathrm{C}++\end{array}$ \\
\hline
\end{tabular}


International Journal of Information Technology, Modeling and Computing (IJITMC) Vol. 4, No.4, November 2016

\begin{tabular}{|c|c|c|c|c|l|}
\hline 7 & $\begin{array}{c}\text { Type Of } \\
\text { Simulation }\end{array}$ & $\begin{array}{c}\text { Parallel Discrete } \\
\text { Event Simulation } \\
\text { (OPNET } \\
\text { Modeler } \\
\text { Wireless suite } \\
\text { version 16.0) }\end{array}$ & $\begin{array}{c}\text { Discrete Event } \\
\text { Simulation }\end{array}$ & $\begin{array}{c}\text { Parallel } \\
\text { Discrete } \\
\text { Event } \\
\text { Simulation }\end{array}$ & $\begin{array}{c}\text { Discrete Event } \\
\text { Simulation }\end{array}$ \\
\hline 8 & Developed By & $\begin{array}{c}\text { Now it is being } \\
\text { taken over by } \\
\text { Riverbed }\end{array}$ & $\begin{array}{c}\text { OMNet++ } \\
\text { Developers Group }\end{array}$ & $\begin{array}{l}\text { DARPA. But } \\
\text { currently } \\
\text { development } \\
\text { being done with } \\
\text { SAMAN and } \\
\text { through NSF } \\
\text { with CONSER } \\
\text { with other } \\
\text { researchers } \\
\text { including ACIRI }\end{array}$ \\
\hline
\end{tabular}

\subsection{NeTWORK SimUlator 2:}

Network Simulator NS-2 (Network Simulator) [8] is a discrete event driven simulator used for implementation and simulations of various network protocols.NS-2 was developed in the year 1989 and it's freely distributed, open source. It is a de facto standard in networking research. NS2 is used for modelling network component like - Transport protocols: TCP and UDP - multicast, ad-hoc routing, Drop tail etc - Link layer: Point to point connection, Local Area Networks, wireless connections used in infrastructure for tracing, visualization, error models and to modify or create our own modules. This tool for implementation of this paper because of its modular and open architecture.

The simulation that's why we have used the NS2 in our project due to easy to learning, user friendly, GUI as well as command line, open source, Linux based also real time and large number source capability.

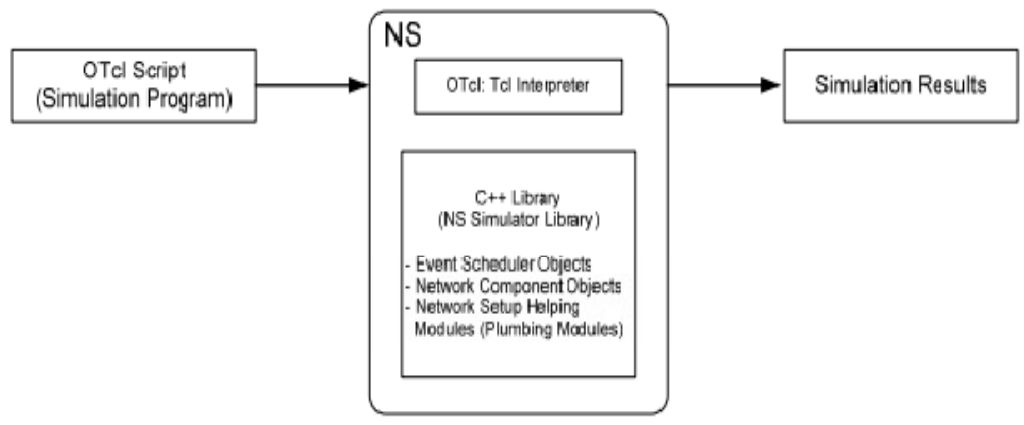

Fig 3. Basic Structure of NS-2 Simulation [8]

NS-2 is totally based on Object Oriented (OO)The simulator framework uses a two-language programming approach, OTcl and $\mathrm{C}++$. OTcl is used for network components and description of simulation scenarios.It is used globally in academia. It can run on Solaris and even windows via use of third party software called Cygwin. The latest version is 2.36 [24]. The earlier versions of network simulator-2 architecture did not support wireless communication; they only supported wired stationary nodes. Later on different research groups added wireless nodes and channels with a focus on wireless ad-hoc networks. The framework allows a very detailed modeling of 
wireless communication using radio prorogation models, Antennas, link layer, ARP, MAC layer protocols (e.g. IEEE 802.11), as well as ad-hoc routing protocols like AODV, DSR, DSDV etc. Simulation Topology and Environment Following simulation parameters were adopted to test the validity of the modified approach $[8,10]$.

\section{Simulation Set UP:}

Exponential traffic voice (created packet while talk period for $1.00 \mathrm{~ms}$ and no packet is created during $1.3 \mathrm{~ms}$ of silent period) is a scenario in the implementation using SIP to fulfill sending voice packet from end to end nodes.

\section{TOPOLOGY}

Considering that, the network topology consists of 1 main base station, along with node for sending voice $\mathrm{n}$ _voice and node for receiving call is $\mathrm{n}$ _null for each server, which means n_voice1, n_voice2, n_null1 and n_null2. In addition, one node for testing, cooperating with each server is attached with sending or receiving node ( $\mathrm{n} \_$test 1 and $\mathrm{n} \_$test 2 ).

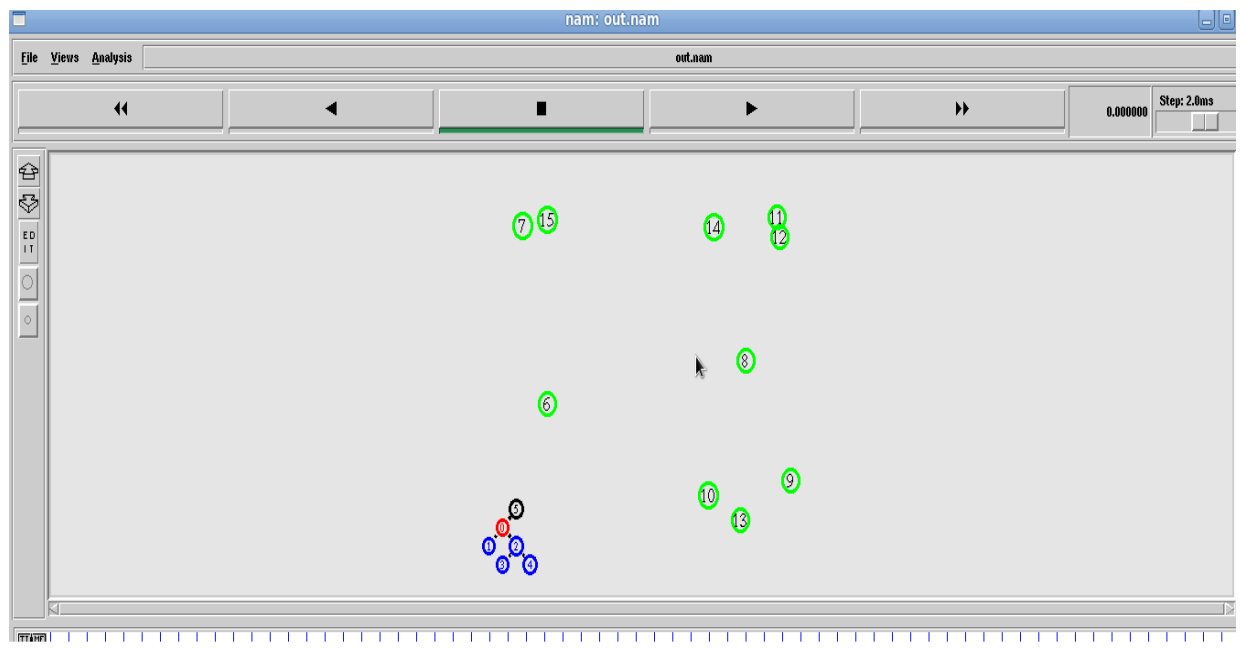

\section{Flow CHART:}

Fig 4. Topology 


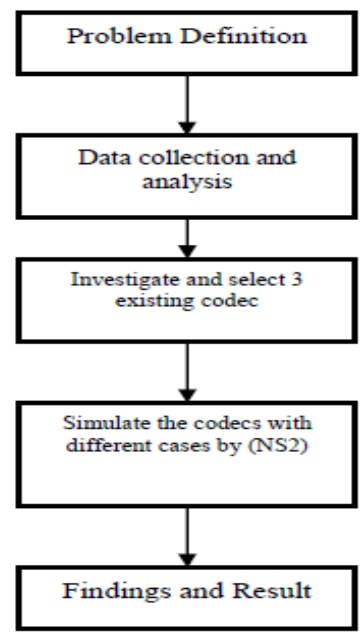

Fig 5. Project Structure Steps

\section{Result And Discussion:}

In this Section, we compare the capabilities of the three VOIP codec's studied in this paper. To evaluate more reliable performance of G.711, G.723 and G.729 VoIP codec's in same simulation environment (50 to 300 mobile nodes). Performance metrics are calculated from trace file, with the help of AWK program. The simulation results shown in the following section in the form of line graph with description. The result shows the comparison between the three codecs on different QoS parameters in a VoIP network with Non-mobility scenarios.

\section{GRAPHS OF NON-MOBILITY SCENARIO IN WI-FI:}

\subsection{NON-ADAPTATION:}

\subsubsection{DELAY}

From the below figure and table Delay was gained at destination node against various dimension of networks and varied the simulation time uniformly for each codec. This data may be delivered over a physical or logical link, or pass through a certain network node. It is clear that G.729 gives less delay when the nodes are less. G.711 and G.723 codecs gives less delay. G.729 had a high delay. From these graphs it is clear that delay increase with increase in non-mobility nodes.

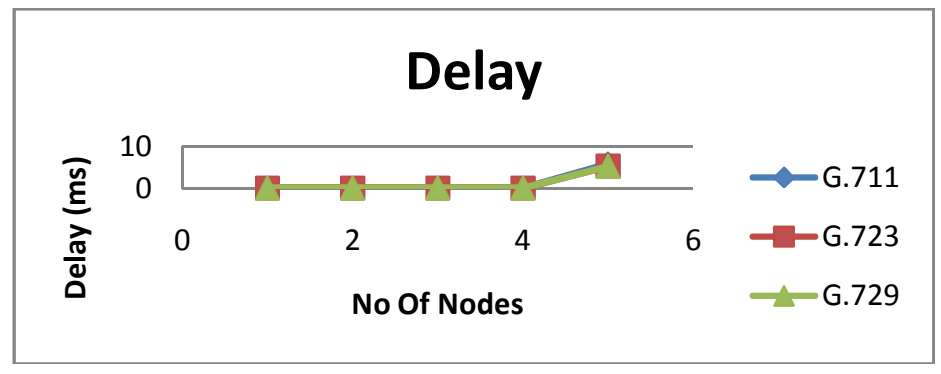

Fig. 6 Delay vs Number of nodes 


\begin{tabular}{|c|c|c|c|}
\hline \multicolumn{4}{|c|}{ Delay } \\
\hline No of Nodes & G.711 & G.723 & G.729 \\
\hline 1 & 0.00517975 & 0.00520132 & 0.00520509 \\
\hline 2 & 0.00611273 & 0.00634488 & 0.00634488 \\
\hline 3 & 0.00632576 & 0.006492 & 0.006492 \\
\hline 4 & 0.0059831 & 0.0061244 & 0.0061244 \\
\hline 5 & 5.70636 & 5.17704 & 5.17704 \\
\hline
\end{tabular}

Table 4:Readings of Delay vs Number of nodes

Average end to end delay metric is shown in fig above G.729 presents the best performance with respect to other codes. These results are due to transfer rate and packet size. The low packets transfer and the larger packet size, the more time is required to process them. The relatively high transfer rate $(8 \mathrm{kbps})$ and low packet size (20 bytes) for G.729 make G.729 makes ideal codecs. Otherwise, G.723 and G.711 suffered highest delay than G.729 for the reason that it has the lowest bit rate (5.3 kbps for G.723) and larger packet size (160 bytes for G.711). In turn, the end to end delay is increased with transfer rate and packet size.

\subsubsection{MOS}

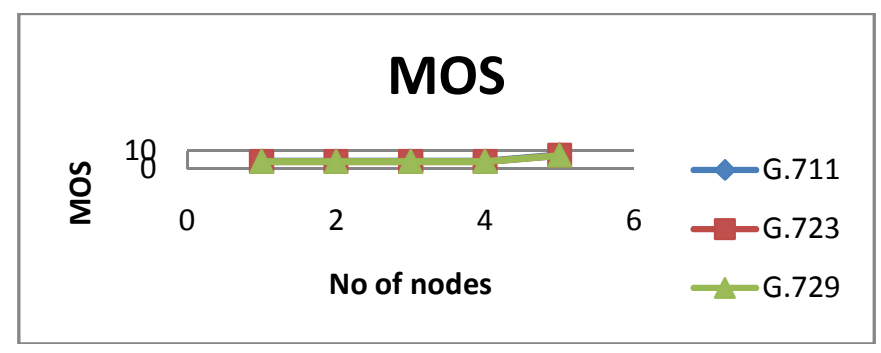

Fig.7 MOS vs Number of nodes

\begin{tabular}{|c|c|c|c|}
\hline \multicolumn{4}{|c|}{ MOS } \\
\hline No of Nodes & G.711 & G.723 & G.729 \\
\hline 1 & 4.1 & 3.9 & 3.92 \\
\hline 2 & 4.1 & 3.9 & 3.92 \\
\hline 3 & 4.1 & 3.9 & 3.92 \\
\hline 4 & 4.1 & 3.9 & 3.92 \\
\hline 5 & 7.71111 & 7.351 & 7.371 \\
\hline
\end{tabular}

Table 5 Readings of MOS vs Number of nodes

The most widely used QoS metric in VOIP applications is MOS. The MOS value describes the voice perception quality. The average MOS value for the three codecs is represented in figure above. Codecs G.711 and G.729 have acceptable MOS value G.723 and G.729, respectively. On the other hand, the MOS value for G.711 is 4.1 which indicate that the quality of service is good if this codec is used.

\subsubsection{Packet Delivery Ratio}


Figure below figure and table describes the average voice PDR comparison using different codecs. From the figure, the variation of the codec is minimum and approximately varying throughout the simulation. The average voice throughput variation in case of codec G.711 is lower than the other two codecs at the earlier time of simulation. The throughput variation in case of G.729 lies between two other audio codecs. So audio codec G.711 gives better results than audio codecs G.723 and G.729 respectively.

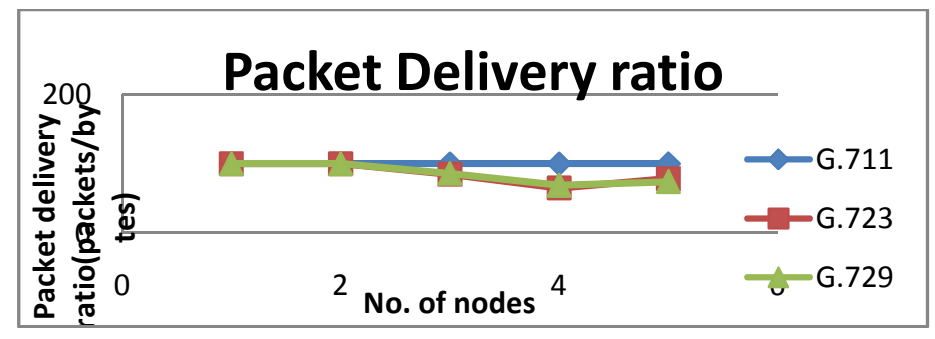

Fig.8 PDR vs Number of nodes

Table 6 Readings of PDR vs Number of nodes

\begin{tabular}{|c|c|c|c|}
\hline \multicolumn{4}{|c|}{ Packet Delivery ratio } \\
\hline No of Nodes & G.711 & G.723 & G.729 \\
\hline 1 & 100 & 100 & 100 \\
\hline 2 & 100 & 100 & 100 \\
\hline 3 & 100 & 84.7495 & 86.105 \\
\hline 4 & 100 & 64.8694 & 69.1432 \\
\hline 5 & 99.9068 & 78.35 & 74.4644 \\
\hline
\end{tabular}

\subsubsection{ThroughPUT}

Figure below figure and table describes the average voice throughput comparison using different codecs. From the figure, the variation of the codec is minimum and approximately varying PDR the simulation. The average voice throughput variation in case of codec G.711 is higher than the other two codecs at the earlier time of simulation. But after some time it falls down. The throughput variation in case of G.723 lies between two other audio codecs. So audio codec G.729 gives better results than audio codecs G.711 and G.723 respectively.

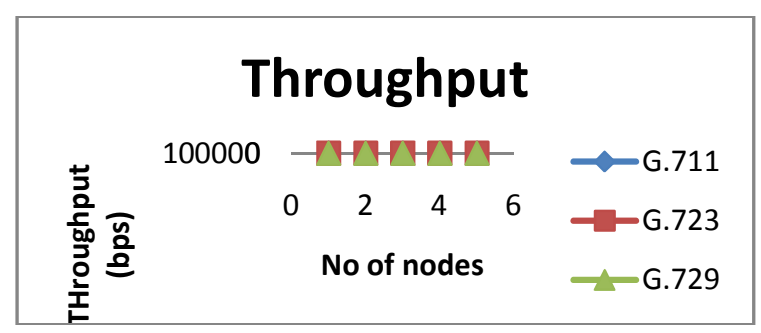

Fig.9 Throughput vs Number of nodes 
International Journal of Information Technology, Modeling and Computing (IJITMC) Vol. 4, No.4, November 2016

\begin{tabular}{|c|c|c|c|}
\hline \multicolumn{4}{|c|}{ Throughput } \\
\hline No of Nodes & G.711 & G.723 & G.729 \\
\hline 1 & 18029.9 & 18588.6 & 19784.5 \\
\hline 2 & 29114.8 & 28454.4 & 28454.4 \\
\hline 3 & 30741 & 28931.3 & 28931.3 \\
\hline 4 & 37863.3 & 38280.2 & 38280.2 \\
\hline 5 & 44130.3 & 50565 & 50565 \\
\hline
\end{tabular}

Table 7 Readings of Throughput vs Number of nodes

\subsubsection{JITTER}

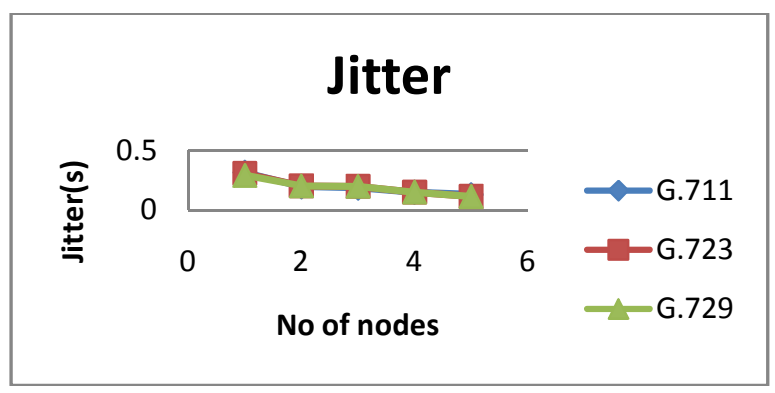

Fig.10 Jitter vs Number of node

Table 8Readings of Jitter vs Number of nodes

\begin{tabular}{|c|c|c|c|}
\hline \multicolumn{4}{|c|}{ Jitter } \\
\hline No of Nodes & G.711 & G.723 & G.729 \\
\hline 1 & 0.3162 & 0.30886 & 0.29013 \\
\hline 2 & 0.195503 & 0.20146 & 0.20146 \\
\hline 3 & 0.185009 & 0.19794 & 0.19794 \\
\hline 4 & 0.150133 & 0.14956 & 0.14956 \\
\hline 5 & 0.128761 & 0.11322 & 0.11322 \\
\hline
\end{tabular}

Figure above figure and table describes the average voice jitter comparison using different codecs. From the figure, the variation of the codec is minimum and approximately constant throughout the simulation. The average voice jitter variation in case of codec G.711 is higher than the other two codecs at the earlier time of simulation. But after some time it falls down. The jitter variation in case of G.723 lies between two other audio codecs. The voice jitter threshold for smooth communication in VOIP network is about $1 \mathrm{~ms}$ so audio codec G.729 gives better results than audio codecs G.711 and G.723 respectively. So there high increase in jitter as audio codecs G.711 and G.723 are added to the network. This increase in voice jitter makes the voice difficult to understand due to arriving packets at different time. The use audio codec G.729 will make the jitter less and best performance of VOIP application in Integrating Wireless LAN.

Average voice traffic sent and received is presented in Figure above. Any network to be more efficiency these two traffic must be equal. The traffic received by the network with codec G.729 is less deviated from the traffic sent comparatively with codec G.723 and G.711. This analysis indicates that the noise added in the G.729 network is less when compared to the other networks, so this codec is more efficient. 


\subsection{ADAPTATION:}

\subsubsection{DELAY}

From the below figure and table Delay was gained at destination node against various dimension of networks and varied the simulation time uniformly for each codec. This data may be delivered over a physical or logical link, or pass through a certain network node. it is clear that G.729 gives more delay when the nodes are more. G.711 and G.723 codecs gives less delay. G.729 had a high delay. From these graphs it is clear that delay decrease with increase in non-mobility nodes.

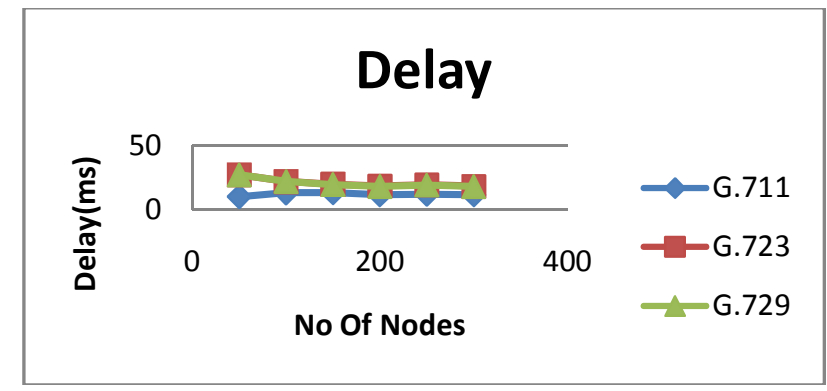

Fig.11 Delay vs Number of nodes

\begin{tabular}{|c|c|c|c|}
\hline \multicolumn{4}{|c|}{ Delay } \\
\hline No of Nodes & G.711 & G.723 & G.729 \\
\hline 50 & 10.006 & 27.1402 & 27.0985 \\
\hline 100 & 12.9204 & 21.8371 & 21.9207 \\
\hline 150 & 12.9789 & 19.7138 & 19.6157 \\
\hline 200 & 11.5245 & 18.402 & 18.0957 \\
\hline 250 & 11.7394 & 19.312 & 19.1733 \\
\hline 300 & 11.4341 & 18.1266 & 18.0227 \\
\hline
\end{tabular}

Table 9 Readings of Delay vs Number of nodes

\subsubsection{Mos}

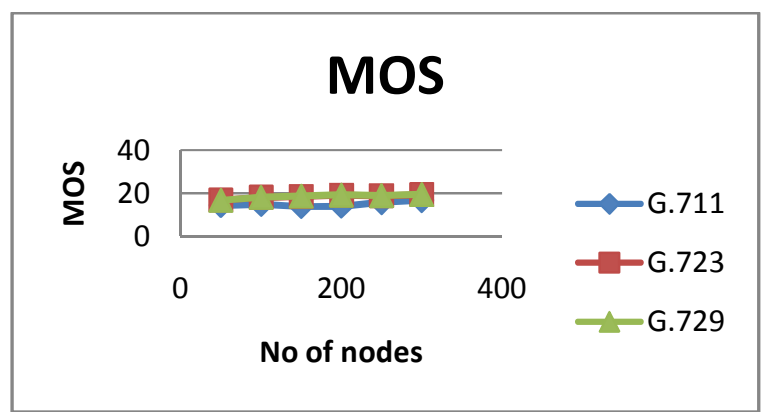

Fig. 12 MOS vs Number of nodes 


\begin{tabular}{|c|c|c|c|}
\hline \multicolumn{4}{|c|}{ MOS } \\
\hline No of Nodes & G.711 & G.723 & G.729 \\
\hline 50 & 14.4266 & 16.8844 & 16.7141 \\
\hline 100 & 14.8738 & 18.1194 & 18.0848 \\
\hline 150 & 13.8938 & 18.5201 & 18.5863 \\
\hline 200 & 14.0479 & 19.086 & 19.1325 \\
\hline 250 & 15.7118 & 18.8408 & 18.8946 \\
\hline 300 & 16.6547 & 19.3664 & 19.3738 \\
\hline
\end{tabular}

Table 10 Readings of MOS vs Number of nodes

The most widely used QoS metric in VOIP applications is MOS. The MOS value describes the voice perception quality. The average MOS value for the three codecs is represented in figure above. From the graph it is observed that MOS value increases as the nodes increases. Codecs G.711 and G.723 have acceptable MOS value G.723 and G.729, respectively. On the other hand, the MOS value for G.729 is 19.3738 which indicate that the quality of service is good if this codec is used.

\subsubsection{PDR}

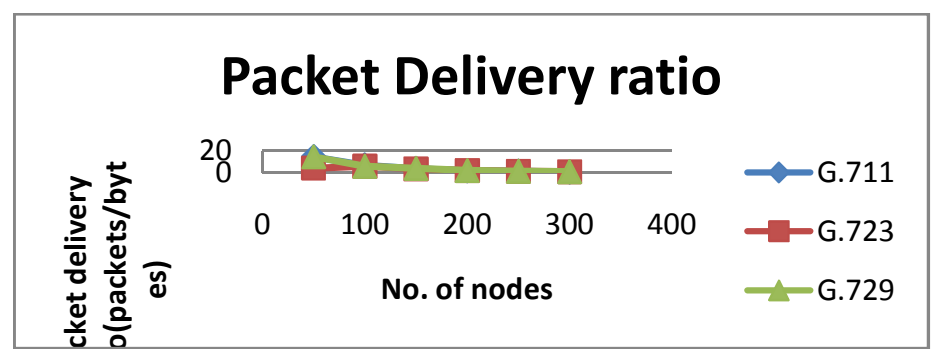

Fig.13 PDR vs Number of nodes

\begin{tabular}{|c|c|c|c|}
\hline & Packet Delivery ratio & & \\
\hline No of Nodes & G.711 & $\mathbf{G . 7 2 3}$ & G.729 \\
\hline 50 & 14.7053 & 4.2897 & 14.4356 \\
\hline 100 & 7.04911 & 6.16613 & 5.97557 \\
\hline 150 & 3.96553 & 3.79713 & 4.18666 \\
\hline 200 & 2.05143 & 2.14277 & 2.0679 \\
\hline 250 & 1.50003 & 1.5714 & 1.53505 \\
\hline 300 & 0.885652 & 1.14652 & 1.17401 \\
\hline
\end{tabular}

Table 11 Readings of PDR vs Number of nodes

Figure above figure and table describes the average voice PDR comparison using different codecs. From the figure, the variation of the codec is minimum after 100 nodes and 
approximately varying throughout the simulation. The average voice throughput variation in case of codec G.723 is lower than the other two codecs at the earlier time of simulation. The PDR variation in case of G.729 lies between two other audio codecs. So audio codec G.723 gives better results than audio codecs G.711 and G.729 respectively.

\subsubsection{THROUGHPUT}

Figure below figure and table describes the average voice throughput comparison using different codecs. From the figure, the variation of the codec is minimum and approximately varying throughout the simulation. The average voice throughput variation in case of codec G.711 is higher than the other two codecs at the earlier time of simulation.But after some time it falls down. The throughput variation in case of G.723 lies between two other audio codecs.

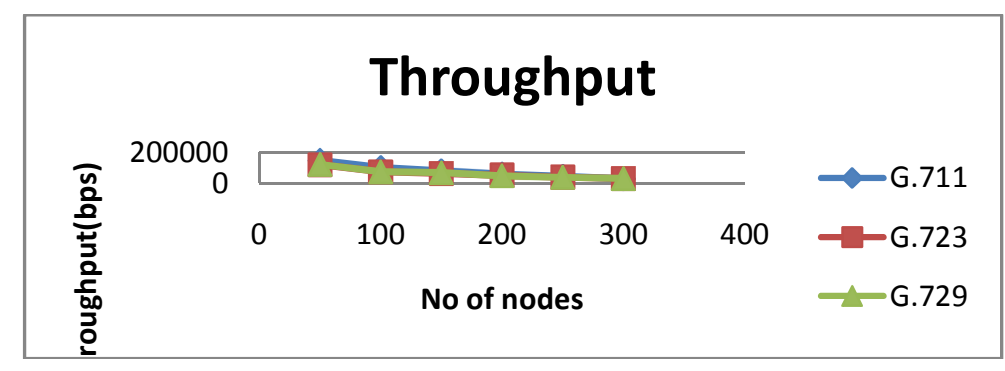

Fig.14 Throughput vs Number of nodes

Table 12 Readings of Throughput vs Number of nodes

\begin{tabular}{|c|c|c|c|}
\hline \multicolumn{4}{|c|}{ Throughput } \\
\hline No of Nodes & G.711 & $\mathbf{G . 7 2 3}$ & G.729 \\
\hline 50 & 146976 & 118617 & 121296 \\
\hline 100 & 101554 & 73229.2 & 73132.1 \\
\hline 150 & 80945.3 & 62387.5 & 67257.9 \\
\hline 200 & 59712.1 & 51735.5 & 48410.9 \\
\hline 250 & 46505.4 & 39101.3 & 38431.8 \\
\hline 300 & 28917.1 & 30617.7 & 30371.3 \\
\hline
\end{tabular}

So audio codec G.729 gives better results than audio codecs G.711 and G.723 respectively.

\subsubsection{JITTER}

From the below figure and table, the variation of the codec is minimum and approximately constant throughout the simulation. The average voice jitter variation in case of codec G.711 is higher than the other two codecs at the earlier time of simulation. But after some time it falls down. The jitter variation in case of G.729 lies between two other audio codecs. The voice jitter threshold for smooth communication in VOIP network is about 1ms so audio codec G.729 gives better results than audio codecs G.711 and G.723 respectively. 
International Journal of Information Technology, Modeling and Computing (IJITMC) Vol. 4, No.4, November 2016

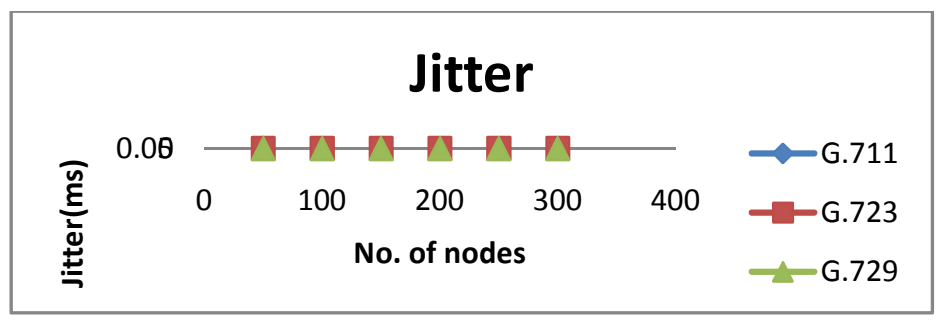

Fig.15 Jitter vs Number of nodes

Table 13 Readings of Jitter vs Number of nodes

\begin{tabular}{|c|c|c|c|}
\hline \multicolumn{4}{|c|}{ Jitter } \\
\hline No of Nodes & G.711 & G.723 & G.729 \\
\hline 50 & 0.00833 & 0.008498 & 0.008088 \\
\hline 100 & 0.007473 & 0.008641 & 0.008145 \\
\hline 150 & 0.008015 & 0.008401 & 0.00785 \\
\hline 200 & 0.009502 & 0.009492 & 0.009489 \\
\hline 250 & 0.012755 & 0.012794 & 0.012794 \\
\hline 300 & 0.019217 & 0.014816 & 0.014902 \\
\hline
\end{tabular}

\section{COMPARISON OF VOIP CODECS.}

\begin{tabular}{|l|c|c|c|c|c|c|c|c|c|}
\hline \multicolumn{4}{|c|}{ Codec Information } & \multicolumn{4}{c|}{ Bandwidth Calculations } \\
\hline $\begin{array}{l}\text { Codec \& Bit } \\
\text { Rate (Kbps) }\end{array}$ & $\begin{array}{c}\text { Codec } \\
\text { Sample } \\
\text { Size } \\
\text { (Bytes) }\end{array}$ & $\begin{array}{c}\text { Codec } \\
\text { Sample } \\
\text { Interval } \\
(\mathbf{m s})\end{array}$ & $\begin{array}{c}\text { Mean } \\
\text { Opinion } \\
\text { Score } \\
\text { (MOS) }\end{array}$ & $\begin{array}{c}\text { Voice } \\
\text { Payload } \\
\text { Size } \\
\text { (Bytes) }\end{array}$ & $\begin{array}{c}\text { Voice } \\
\text { Payload } \\
\text { Size } \\
\text { (ms) }\end{array}$ & $\begin{array}{c}\text { Packets } \\
\text { Per } \\
\text { Second } \\
\text { (PPS) }\end{array}$ & $\begin{array}{c}\text { Bandwidth } \\
\text { MP or } \\
\text { FRF.12 } \\
\text { (Kbps) }\end{array}$ & $\begin{array}{c}\text { Bandwidth } \\
\text { w/cRTP } \\
\text { MP or } \\
\text { FRF.12 } \\
\text { (Kbps) }\end{array}$ & $\begin{array}{c}\text { Bandwidth } \\
\text { Ethernet } \\
\text { (Kbps) }\end{array}$ \\
\hline $\begin{array}{l}\text { G.711 (64 } \\
\text { Kbps) }\end{array}$ & $\begin{array}{c}80 \\
\text { Bytes }\end{array}$ & $10 \mathrm{~ms}$ & 4.1 & $\begin{array}{c}160 \\
\text { Bytes }\end{array}$ & $20 \mathrm{~ms}$ & 50 & $82.8 \mathrm{Kbps}$ & $67.6 \mathrm{Kbps}$ & $87.2 \mathrm{Kbps}$ \\
\hline G.729(8 Kbps) & $\begin{array}{c}10 \\
\text { Bytes }\end{array}$ & $10 \mathrm{~ms}$ & 3.92 & 20 Bytes & $20 \mathrm{~ms}$ & 50 & $26.8 \mathrm{Kbps}$ & $11.6 \mathrm{Kbps}$ & $31.2 \mathrm{Kbps}$ \\
\hline $\begin{array}{l}\text { G.723.1(6.3 } \\
\text { Kbps) }\end{array}$ & $\begin{array}{c}24 \\
\text { Bytes }\end{array}$ & $30 \mathrm{~ms}$ & 3.9 & 24 Bytes & $30 \mathrm{~ms}$ & 33.3 & $18.9 \mathrm{Kbps}$ & $8.8 \mathrm{Kbps}$ & $21.9 \mathrm{Kbps}$ \\
\hline $\begin{array}{l}\mathrm{G} .723 .1(5.3 \\
\text { Kbps) }\end{array}$ & $\begin{array}{c}20 \\
\text { Bytes }\end{array}$ & $30 \mathrm{~ms}$ & 3.8 & 20 Bytes & $30 \mathrm{~ms}$ & 33.3 & $17.9 \mathrm{Kbps}$ & $7.7 \mathrm{Kbps}$ & $20.8 \mathrm{Kbps}$ \\
\hline
\end{tabular}

Table 14 Comparison of different Codecs

\section{CONCLUSION:}

In this project, we evaluated the performance of three different VoIP codecs over Wi-Fi networks. The VoIP performance is measured in three scenarios using NS2 MOS, jitter, and average end-toend delay are analysed as performance parameters which define QoS in VoIP. G. 711 codec showed the better codec for VoIP over Wi-Fi. 


\section{FUTURE WORK:}

This project considered voice calls from fixed nodes. The impact of mobility on VoIP performance is suggested as future research. Also, for future study, various codecs must be investigated on Wi-Fi networks to observe the performance of VoIP and the QoS parameters should be improved to get the maximum throughput and PDR, minimum delay and jitter.

\section{REFERENCES:}

[1] A. M. Noll, ICybernetwork technology: issues and uncertainties," Commun. ACM, vol. 39, pp. $27\{31$, dec 1996.

[2] A survey of quality of service in IEEE802.11 Networks,HUA ZHU, MING LI, IMRICH CHLAMTAC, AND B. PRABHAKARANTHE UNIVERSITY OF TEXAS AT DALLAS.

[3] A new method for VoIP Quality of Service control using combined adaptive sender rate and priority marking, ZizhiQiao, Lingfen Sun, Nicolai Heilemann and Emmanuel Ifeachor.

[4] Adaptive QoS Control Approaches for VoIP Over Multi-Rate WLANs, H. Kazemitabar, A. Md. Said UniversitiTeknologi PETRONAS Bandar Seri Iskandar, 31750 Tronoh, Perak, Malaysia.

[5] E. Haghani and N. Ansari, IVoip traffic scheduling in wimax networks," in Global Telecommunications Conference, 2008. IEEE GLOBECOM 2008. IEEE, pp. 1 \{5, 30 2008-dec. 4 2008.

[6] H. Kazemitabar, A. Md. Said UniversitiyTechnology PETRONAS hanieh80@gmail.com Adaptive QoS Control Approaches for VoIP Over Multi-Rate WLAN's.

[7] I. Adhicandra, Measuring data and voip traffic in wimax networks," CoRR, vol. abs/1004.4583, 2010.

[8] ITU-T

[9] J. Yu and I. Al-Ajarmeh, ICall admission control and traffic engineering of voip," in Digital Telecommunications, 2007. ICDT '07. Second International Conference on, p. 11, july 2007.

[10] M. I. Tariq, M. A. Azad, R. Beuran, and Y. Shinoda, IPerformance analysis of voip codecs over be wimax network," 3rd International Conference on Computer and Electrical Engineering (ICCEE 2010), pp. 47\{51, November 2010.

[11] M. A. Qureshi, A. Younus, M. Saeed, F. A. Sidiqui, N. Touheed, and M. S. Qureshi, IComparative study of voip over wimax and wi_," International Journal of Computer Science, vol. 8, pp. 433\{437, May 2011.

[12] Priority Based Dynamic Rate Control for VoIP Traffic, Fariza Sabrina CSIRO ICT Centre, Sydney, Australia.

[13] Performance Analysis of VoIP Codecs over WiMAX, Khaled Alutaibi

[14] Performance Analysis Of VOIP Codec's With QoS, Parameters, Priyanka Grover1, Meenakshi Chawla2 Dept. of Computer Engineering, TIT\&S, Bhiwani.

[15] PERFORMANCE ANALYSIS OF VOIP TRAFFIC OVER INTEGRATING WIRELESS LAN AND WAN USING DIFFERENT CODECS, Ali M. Alsahlany.

[16] Performance Analysis of various Codecs Schemes of VOIP over WiMAX, M-Tech Student\#1, Assit. Prof. *2 \& Dept. of CSE., ManavRachna International University, Faridabad, Haryana, India.

[17] Performance Analysis of VOIP Codes under Different Mobility Pattern,Manpreet Kaur.

[18] Performance Evaluation of The Quality of VoIP Over WLAN Codecs, H.A. Ifijeh, F.E. Idachaba, and I.B. Oluwafemi

[19] R. S. Ramakrishnan and P. V. kumar, IPerformance analysis of different codecs in voip using sip," in Mobile and Pervasive Computing (CoMPC2008), August 2008.

[20] S. Islam, M. Rashid, and M. Tarique, VArticle: Performance analysis of WiMax/WiFi system under different codecs," International Journal of Computer Applications, vol. 18, pp. 13\{19, March 2011. Published by Foundation of Computer Science.

[21] S. Jadhav, H. Zhang, and Z. Huang, IPerformance evaluation of quality of voip in wimax and umts.," in PDCAT, pp. 375\{380, IEEE Computer Society, 2011. 
International Journal of Information Technology, Modeling and Computing (IJITMC) Vol. 4, No.4, November 2016

[22] V. Toncar, IVoip basics: Overview of audio codecs," April 2012. Available: http://toncar.cz/Tutorials/VoIP/VoIP Basics Overview of Audio Codecs.html. Voip codecs," April 2012. Available: http://www.voip-sip.org/voip-codecs.

[23] VoIP codec adaptation algorithm in multirate 802.11 WLANs, Anna Sfairopoulou, Carlos Macilan, Boris Bellalta.

[24] VoIP over Wireless LAN Survey, RăzvanBeuran,Internet Research Center Japan Advanced Institute of Science and Technology (JAIST).

\section{AUTHORS}

Shreekant Gurrapu is working as Network Architect in Tech Mahindra Limited, India and I am student of VIT, Engineering College Mumbai, India. My research is focus on Performance Analysis of VOIP codec's over WLAN, Network management.

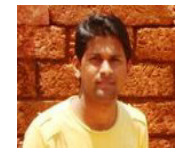

Dr. Saurabh Mehta is Professor and Head Department of EXTC, VIT Engineering College Mumbai, India and his area of specializations includes a core wireless network, Sensor Network. His wireless and NS2 ideas helps me lot in this research paper.

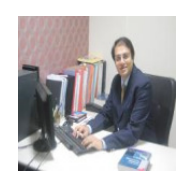

Prof. Shraddha Panbude is working in VIT Engineering College Mumbai, India and she is my guide of Master's project.

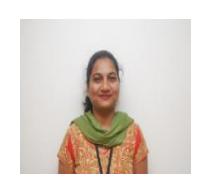

